\title{
Energy injection in pre-recombination era and EDGES detection
}

To cite this article: Nirmalya Brahma et al JCAP12(2020)034

View the article online for updates and enhancements.

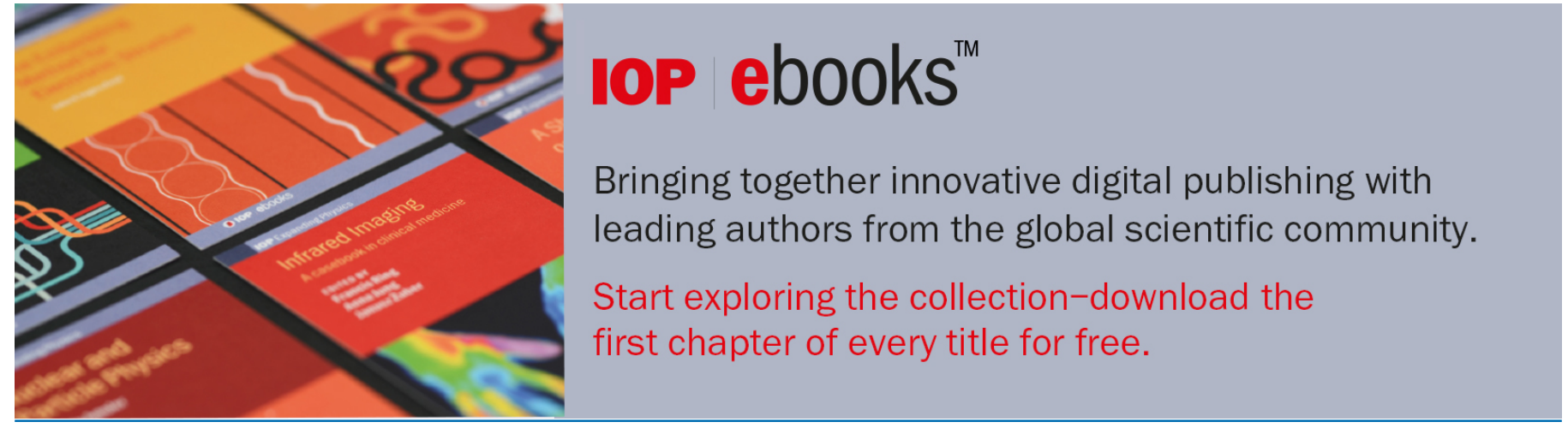

This content was downloaded from IP address 14.139 .128 .34 on $25 / 01 / 2021$ at 07:10 


\title{
Energy injection in pre-recombination era and EDGES detection
}

\author{
Nirmalya Brahma, ${ }^{a, 1}$ Shiv Sethi ${ }^{b}$ and Shivnag Sista ${ }^{a}$ \\ ${ }^{a}$ Indian Institute of Science, \\ C.V.Raman Avenue, Bangalore, Karnataka 560012, India \\ ${ }^{b}$ Raman Research Institute, \\ C.V.Raman Avenue, Bangalore, Karnataka 560080, India \\ E-mail: nirmalyab@iisc.ac.in, sethi@rri.res.in, shivnag@iisc.ac.in
}

Received July 14, 2020

Revised September 15, 2020

Accepted October 31, 2020

Published December 21, 2020

\begin{abstract}
We study the possibility of explaining the recent EDGES detection by an energy injection in the pre-recombination era. Our aim is to show that the residue of this energy injection could give the resultant increase in the energy density at frequencies $x_{e} \equiv h \nu /(k T) \simeq$ $10^{-3}$, which is needed to explain the EDGES result. We consider two models of energy injection: Gaussian profile with a fixed redshift of injection and radiative decay of a nonrelativistic particle. We show that the energy injection should occur in the redshift range $z \lesssim 4 \times 10^{3}$ to prevent free-free processes from thermalizing the injected energy. The injected energy should be nearly $200-1000$ times the CMB intensity at $x_{e} \simeq 10^{-3}$ to obtain the requisite residue. A large fraction of the injected energy gets thermalized and therefore distorts the CMB spectrum. We compute CMB spectral distortion for both the models and show that the fractional change in CMB energy density, $\Delta \rho_{\mathrm{CMB}} / \rho_{\mathrm{CMB}} \simeq 10^{-6}$, which might be detectable with the proposed experiment PIXIE. We also outline the implication of our proposed scenario for $\mathrm{CMB}$ anisotropies.
\end{abstract}

Keywords: CMBR theory, physics of the early universe, reionization

ArXiv ePrint: 2007.06417

\footnotetext{
${ }^{1}$ Corresponding author.
} 


\section{Contents}

1 Introduction 1

2 HI signal, EDGES detection, and possible explanations 2

3 CMB spectrum: physical processes 4

4 Analytical approach and approximations $\quad 6$

5 Numerical solution of Kompaneets equation $\quad 9$

$\begin{array}{lll}5.1 & \text { Decaying particle } & 11\end{array}$

5.2 Explaining EDGES data 12

6 CMB spectral distortion $\quad 13$

$\begin{array}{lll}\text { 6.1 Impact on CMB anisotropies } & 14\end{array}$

7 Summary and conclusions $\quad 15$

$\begin{array}{ll}\text { A Time scales of various radiative processes } & 16\end{array}$

\section{Introduction}

In recent years, major advances have been made in our understanding of the formation of structures in the universe via precise cosmic microwave background (CMB) anisotropy experiments [1-3] and large scale structure surveys [4]. Another major cornerstone of modern cosmology is the near-blackbody spectrum of CMB. The COBE-FIRAS experiment gave the current upper bounds on the CMB spectral distortion parameters: $|\mu| \lesssim 9 \times 10^{-5}$ and $|y| \lesssim 1.5 \times 10^{-5}[5]$. Such stringent upper limits on the deviation of CMB spectrum from a blackbody put strong constraints on processes that inject energy into the plasma in the universe after $z \simeq 10^{6}$ (for details e.g. [6-22]). The proposed experiment PIXIE [23] will improve FIRAS limits by many orders of magnitude: $y \simeq 10^{-9}$ and $\mu \simeq 10^{-8}$.

CMB physics has also enhanced our understanding of the dark age of the universe which is predicted to have ended around redshift $z \simeq 35$ with the formation of first largescale structures (epoch of cosmic dawn). This era was followed by the epoch of reionization (EoR) during which the UV photons from these collapsed structures emitted radiation which heated and ionized their surrounding medium until $z \simeq 8$ ([24-27]). The CMB temperature and polarization anisotropy detection by WMAP and Planck determine the redshift of reionization, $z_{\text {reion }}=7.75 \pm 0.73([1-3,28-30])$.

The cleanest probe of the physics of EoR is through the detection of redshifted hyperfine $21 \mathrm{~cm}$ line of neutral hydrogen (HI). This signal carries crucial information about the first sources of radiation in the universe and their spectrum in three frequency bands: ultraviolet (UV) radiation (ionizes the surrounding medium), Lyman- $\alpha$ radiation (determines the relative population of neutral hydrogen atoms in hyperfine states), and x-ray photons (heat and partially ionize the medium). In addition, the sources that emitted soft radio photons would also affect the observable HI signal (e.g. [31, 32]). 
The epochs of cosmic dawn and EoR have been studied in detail using numerical, semi-analytic and, analytic methods (e.g. [33-43]). Theoretical estimates based on standard thermal and ionization history suggest the global signal is observable in both absorption and emission with strength $-200-20 \mathrm{mK}$ in a frequency range $50-150 \mathrm{MHz}$, corresponding to a redshift range $25>z>8$ (e.g. [44-47]). The fluctuating component of the signal is expected to be an order of magnitude smaller on scales in the range $3-100 \mathrm{Mpc}$, which implies angular scales $\simeq 1-30$ arc-minutes (e.g. [33, 48-50]; for comprehensive reviews see e.g. [25-27]).

The recent detection of a broad global absorption trough of strength $500 \mathrm{mK}$ by the EDGES group ([51]) at $\nu \simeq 80 \pm 10 \mathrm{MHz}$ is the only positive detection of $\mathrm{HI}$ signal at high redshifts. This detection is in disagreement with the standard scenario and might be pointing to the presence of unknown physics in pre- or post-recombination universe (e.g. milli-charged dark matter or strong radio background at $z \simeq 20[31,32,52-55])$. Such a feature could also potentially be explained by absorption in the Galactic interstellar medium [56].

In this paper, we attempt to explain the EDGES results by an injection of energy in the Rayleigh-Jeans part of the CMB spectrum in the pre-recombination era (for a detailed discussion see [57]). If the injected photons equilibrate before the recombination occurs, there would be no trace of the injection event except global distortion of CMB spectrum. However, depending on the amount of injected energy and the time scale of equilibration, there could be residue excess which might leave observable signatures on the CMB spectrum close to energies of injection. This excess can explain the depth of EDGES' absorption feature. The possibility of such a signature depends on the competition between the rates of interaction between the photon and matter field, the expansion rate, the frequency range, and redshifts at which the photons are injected. In this paper, we address this question by evolving the CMB spectrum after such an energy injection event, using both analytical and numerical methods.

One distinct advantage of studying an energy injection during this era is that both CMB anisotropies and spectral distortion strongly constrain the scope of this injection. Therefore, a generic episode of such energy injection would, in addition to explaining the EDGES detection, likely leave detectable signatures on CMB.

In the next section, we review the HI signal from the EoR and cosmic dawn, the EDGES results, and its proposed explanation. In section 3 we discuss the physical processes involved in determining the CMB spectrum; the relevant time scales are given in the appendix. In section 4, we discuss analytic approaches to solving the coupled evolution of the photon occupation number (Kompaneets equation) and electron temperature. In section 5, the numerical solutions are presented along with the particle decay model. In the final section, we summarize our findings and outline future prospects. Throughout this paper, we assume the spatially-flat $\Lambda \mathrm{CDM}$ model with the following parameters: $\Omega_{m}=0.310, \Omega_{B}=0.049$, and $h=0.677([1])$.

\section{HI signal, EDGES detection, and possible explanations}

In the atom rest frame, hyperfine splitting of the ground state of neutral hydrogen (HI) causes an energy difference that corresponds to a wavelength $\lambda=21.1 \mathrm{~cm}$. The excitation temperature of this line, $T_{S}$, is determined by three processes in the early universe: emission and absorption of CMB radiation which is a blackbody of temperature $T_{\mathrm{CMB}}$, collisions with atoms, and the mixing of the two levels caused by Lyman- $\alpha$ photons (Wouthuysen-Field effect [58-60]). $T_{S}$ can be expressed in terms of the colour temperature of Lyman- $\alpha$ photons, 
$T_{\alpha}$, gas kinetic temperature $T_{K}$, and $T_{\mathrm{CMB}}([26,59,60])$ :

$$
T_{S}=\frac{T_{\mathrm{CMB}}+y_{\alpha} T_{\alpha}+y_{c} T_{K}}{1+y_{\alpha}+y_{c}}
$$

Here $y_{c} \propto n_{\mathrm{H}}$ and $y_{\alpha} \propto n_{\alpha}\left(n_{\mathrm{H}}\right.$ and $n_{\alpha}$ are the number densities of neutral hydrogen atoms and Lyman- $\alpha$ photons, respectively) determine the efficiency of collisions and Lyman$\alpha$ photons, respectively. In the early universe, $1000<z<100, T_{S}$ relaxes to $T_{\mathrm{CMB}}$. In the redshift range $100<z<30$, collisions determine the spin temperature and $T_{S}$ relaxes to the kinetic temperature $T_{K}$ of the matter. As the epoch of reionization commences, the production of Lyman- $\alpha$ photons couples the spin temperature to the colour temperature of Lyman- $\alpha, T_{\alpha}$. It can be shown that multiple scattering of Lyman- $\alpha$ photons with HI causes $T_{\alpha}$ to relax to the kinetic temperature (e.g. [61-63]). Therefore if $y_{\text {tot }}=y_{c}+y_{\alpha} \gtrsim T_{\mathrm{CMB}} / T_{K}$, then $T_{S} \simeq T_{K}$. Otherwise, it relaxes to $T_{\mathrm{CMB}}$.

The HI emits or absorbs 21-cm radiation from CMB depending on whether its excitation temperature $T_{S}$ is greater than or less than $T_{\mathrm{CMB}}$. This temperature difference is observable and, averaged over the sky, can be expressed as (e.g. [26, 44, 46, 47, 64]):

$$
\begin{aligned}
\Delta T_{b} & \simeq \frac{\tau}{1+z}\left(T_{S}-T_{\mathrm{CMB}}\right) \\
& \simeq 26.25 f_{\mathrm{HI}}\left(1-\frac{T_{\mathrm{CMB}}}{T_{S}}\right)\left(\frac{1+z}{10} \frac{0.14}{\Omega_{m} h^{2}}\right)^{\frac{1}{2}}\left(\frac{\Omega_{b} h^{2}}{0.022}\right) \mathrm{mK}
\end{aligned}
$$

Here $f_{\mathrm{HI}}$ is the fraction of gas in the neutral phase.

Recent EDGES observation ([51]) reported a sky-averaged absorption feature of strength $\Delta T \simeq-500 \mathrm{mK}$ in the frequency range $70-90 \mathrm{MHz}$, corresponding to a redshift range 15-19 for the redshifted HI line. It can be shown that, for standard recombination and thermal history, the minimum temperature of the gas at $z \simeq 19$ is $T_{K} \simeq 6 \mathrm{~K}$. It follows from eqs. (2.1) and (2.2) that the absorption trough should not have been deeper than $-180 \mathrm{mK}$.

One possible explanation of the EDGES result is additional radio background in the redshift range $15<z<19$ whose brightness temperature $T_{\text {radio }}$ is higher than the CMB temperature; in this case we can replace $T_{\mathrm{CMB}}$ with the $T_{\mathrm{CMB}}+T_{\text {radio }}$ in eq. $(2.1)$ in the relevant redshift range $([31,32,65])$. With this replacement and suitable choice of $T_{\text {radio }}$ the EDGES result can be explained.

Another plausible explanation invokes the additional cooling of baryons owing to interaction between dark matter and baryons [52]. In this case, we can explain the EDGES detection using eqs. (2.1) and (2.2) if the dark matter-baryon coupling can cool the baryons such that the matter temperature $T_{K} \lesssim 2.5 \mathrm{~K}$ in the redshift range of interest. ${ }^{1}$ Other possible explanations of this result include a possible systematic error [66] and absorption from spinning dust grains in the Galactic ISM [56].

In summary, the EDGES result can be explained by raising $T_{\mathrm{CMB}}$ or reducing $T_{S}$ by a factor of roughly 2.5 in eq. (2.2). Given the uncertainty in other physical processes such as Lyman- $\alpha$ coupling and x-ray heating, this factor provides a lower limit on the required enhancement/reduction in the relevant frequency range (e.g. [43]).

\footnotetext{
${ }^{1}$ EDGES detection implies a sharp trough in the signal at $z \simeq 19$ and a sharp rise at $z \simeq 15$. As the noise level for the detection is $\simeq 20 \mathrm{mK}$ [51], the trough at higher redshift can arise from complete Lyman- $\alpha$ coupling being established close to $z \simeq 19$ with the rapid heating being responsible for the sharp rise at smaller redshift (e.g. [43]).
} 
In this paper, we invoke pre-recombination physics to explain the EDGES result. In particular, we note that the excess radio background needed to explain the EGDES result could be a relic of the pre-recombination era.

We consider energy injection of suitable amplitude and frequency range to explain the EGDES result. In the pre-recombination era, these photons are subject to multiple physical processes which can upscatter (inverse Compton scattering), downscatter (Compton scattering), or absorb (free-free or double Compton processes) these photons. We study the impact of all these processes to discern the range of redshifts at which these photons can be injected. We also study the impact of these photons on other observables such as spectral distortion of $\mathrm{CMB}$ and $\mathrm{CMB}$ anisotropies. In the next section, we review these physical processes.

\section{CMB spectrum: physical processes}

We assume that the energy is injected in a narrow range of frequencies into CMB at an early epoch of the universe. Our aim is to study how this distorted spectrum evolves with time. The redshift range of injection for our study is $10^{3}<z<10^{5}$. The reason for this choice has been elaborated upon in later sections. We need to understand the relative importance of various radiative processes that are responsible for the time evolution of the spectrum.

The photon occupation number $\eta(\nu, t)$ is a function of the frequency (or equivalently, the energy) and time. The following physical processes impact the evolution of the photon occupation number:

- Compton and inverse Compton scattering

- Double Compton emission and absorption

- Free-free emission and absorption

Compton scattering (and its inverse process) only change the energy of photons without affecting the photon number while the other two processes can absorb and create photons.

The rate of change of the photon occupation number due to Compton and inverse Compton scattering off non-relativistic electrons is given by Kompaneets equation ([67-71]):

$$
\left(\frac{\partial \eta}{\partial t}\right)_{C}=\frac{a_{c}}{x_{e}^{2}} \frac{\partial}{\partial x_{e}}\left(x_{e}^{4}\left[\frac{\partial \eta}{\partial x_{e}}+\eta+\eta^{2}\right]\right)
$$

Here $x_{e}=h \nu / k T_{e}$ is a dimensionless parameter, independent of redshift for equilibrium $\eta$, expressed in terms of the physical (not comoving) frequency $\nu$ and the electron temperature $T_{e} ;{ }^{2} a_{c}=n_{e} \sigma_{T} c\left(k T_{e} /\left(m_{e} c^{2}\right)\right)$, where $n_{e}$ is the number density of electrons, $\sigma_{T}$ is the Thompson cross-section and $m_{e}$ is the mass of an electron. The $\partial \eta / \partial x_{e}$ term in the equation corresponds to the contribution for inverse Compton scattering, which results in photons gaining energy from heated electrons. The $\eta$ and $\eta^{2}$ terms on the right-hand side of the equation correspond to spontaneous and stimulated scattering, respectively, both of which contribute to the cooling of photons and are collectively referred to as Compton scattering.

\footnotetext{
${ }^{2}$ The electron temperature is the same as matter temperature throughout the pre-recombination era as the physical processes that equilibrate energy between electrons and baryons act on time scales far shorter than the expansion time scale.
} 
The time evolution of the photon occupation number from double Compton processes - emission, absorption, and stimulated emission — is given by:

$$
\left(\frac{\partial \eta}{\partial t}\right)_{D C}=\frac{C(t)}{x_{e}^{3}}\left[1-\eta\left(\exp \left(x_{e}\right)-1\right)\right]
$$

Here $C(t)=(4 \alpha /(3 \pi))\left(k T_{e} /\left(m c^{2}\right)\right)^{2}\left(I(t) / t_{c}\right), \alpha=e^{2} / \hbar c$ is the fine structure constant and $t_{c}=\left(n_{e} \sigma_{\mathrm{T}} c\right)^{-1}$ is the photon-electron collision time.

$$
I(t)=\int_{0}^{\infty} x_{e}^{4}(1+\eta) d x_{e}
$$

We follow the prescription of [72] in estimating $I(t)$, which gives us $I(t) \simeq 4 \pi^{4} / 15$ (for more discussion on this approximation see [73]).

Similarly, the contribution of all the relevant free-free processes to the evolution of photon occupation number is given by ([68-70]):

$$
\left(\frac{\partial \eta}{\partial t}\right)_{B}=K_{0} \frac{g\left(x_{e}\right) \exp \left(-x_{e}\right)}{x_{e}^{3}}\left(1+\eta\left(x_{e}\right)\left(1-\exp \left(x_{e}\right)\right)\right)
$$

where $g\left(x_{e}\right)$ is the Gaunt factor. For $x_{e} \ll 1, g\left(x_{e}\right) \simeq \sqrt{3} / \pi \ln \left(2.25 / x_{e}\right)$ (e.g. [6]; for a detailed discussion on the Gaunt factor see e.g. [74]). The expression for $K_{0}$ is given in eq. (A.3).

Combining the contributions from Compton, double Compton and free-free processes, we obtain the generalized Kompaneets equation:

$$
\begin{aligned}
\left(\frac{\partial \eta}{\partial t}\right)= & \frac{a_{c}}{x_{e}^{2}} \frac{\partial}{\partial x_{e}}\left(x_{e}^{4}\left[\frac{\partial \eta}{\partial x_{e}}+\eta+\eta^{2}\right]\right)+\frac{C(t)}{x_{e}^{3}}\left[1-\eta\left(\exp \left(x_{e}\right)-1\right)\right] \\
& +K_{0} \frac{g\left(x_{e}\right) \exp \left(-x_{e}\right)}{x_{e}^{3}}\left(1+\eta\left(1-\exp \left(x_{e}\right)\right)\right)
\end{aligned}
$$

This equation is solved along with the evolution of the temperature, $T_{e}$, which is the temperature shared by all the baryons in the pre-recombination era (e.g. [6]):

$$
\frac{d T_{e}}{d t}=-\frac{2 \dot{a}}{a} T_{e}+\frac{\left(T_{\mathrm{eq}}-T_{e}\right)}{t_{e \gamma}}+\frac{q_{\mathrm{ff}}+q_{\mathrm{dc}}}{3 n_{e} k}
$$

Here $t_{e \gamma}=\left(3 m_{e} c / 4 \sigma_{T} \rho_{\gamma}\right)$ and $T_{\text {eq }}$, the effective temperature for an arbitrary photon occupation number $\eta$, is $([75,76])$ :

$$
T_{\text {eq }}=\frac{h \int_{0}^{\infty} d \nu \nu^{4} \eta(\eta+1)}{4 k \int_{0}^{\infty} d \nu \nu^{3} \eta}
$$

$q_{\text {ff }}$ and $q_{\mathrm{dc}}$ give the heating/cooling rates of the medium owing to free-free and double Compton processes:

$$
\begin{aligned}
q_{\mathrm{ff}} & =\frac{8 \pi\left(k T_{e}\right)^{3}}{c^{3} h^{2}} K_{0} \int_{0}^{\infty} g\left(x_{e}\right) \exp \left(-x_{e}\right)\left[1+\eta\left(1-\exp \left(x_{e}\right)\right)\right] d \nu \\
q_{\mathrm{dc}} & =\frac{8 \pi\left(k T_{e}\right)^{3}}{c^{3} h^{2}} C(t) \int_{0}^{\infty}\left[1-\eta\left(\exp \left(x_{e}\right)-1\right)\right] d \nu
\end{aligned}
$$


Before the energy is injected, the baryons and photons share a common temperature with equilibrium photon occupation number given by the Planckian:

$$
\eta\left(x_{e}\right)=\frac{1}{\exp \left(x_{e}\right)-1}
$$

It can readily be checked that for this form of equilibrium photon occupation number the right hand sides of eqs. (3.5) and (3.6) vanish ${ }^{3}$ and eq. (3.7) yields $T_{\mathrm{eq}}=T_{e}$. In an expanding universe, eq. (3.10) is left unchanged as $x_{e}$ is an invariant at early times. After the injection of energy, $x_{e}$ is not an invariant and we can choose another variable which remain invariant during the expansion of the universe (see below).

The time scales of the physical processes discussed above are given in the appendix.

\section{Analytical approach and approximations}

Our aim is to solve eqs. (3.5) and (3.6) simultaneously after the injection of photons in a narrow frequency range at $x_{e} \ll 1$. We present numerical solutions in the next section. Many of the results we find in this and the next section can also be obtained by an alternative approach developed by Chluba [57]. In this section, we seek an analytic approach based on the physical setting we propose in this paper. Its salient points can be summarized as:

1. The energy is injected in a frequency range for which $x_{e} \ll 1$. To explain the EDGES detection we require a residue of the energy injection in the range: $x_{e} \simeq 1.2-1.6 \times 10^{-3}$.

2. The amount of injected energy is much smaller than the energy density of the CMB, $\delta \rho_{\gamma} \ll \rho_{\mathrm{CMB}}$. If the energy is injected for a range of frequencies between $x_{f} \equiv h \nu_{f} /(k T)$ and $x_{i}$ such that $x_{f} \ll 1$, then

$$
\delta \rho_{\gamma} \simeq \frac{8 \pi(k T)^{4}}{3 c^{3} h^{3}}\left(x_{f}^{3}-x_{i}^{3}\right)\left(\frac{T_{B}}{T}\right)
$$

Here $T_{B}$ is the brightness temperature of the injected photons and $T$ refers to the equilibrium matter/radiation temperature. The CMB energy density, $\rho_{\mathrm{CMB}}=a T^{4}$, where $a=8 \pi^{5} k^{4} /\left(15 h^{3} c^{3}\right)$ is the radiation constant. For $x_{f}=2 \times 10^{-3}, \delta \rho_{\gamma} \simeq$ $\left(T_{B} / T\right) x_{f}^{3} \rho_{\mathrm{CMB}} \simeq 10^{-9} \rho_{\mathrm{CMB}}$ for $T_{B}=T$.

3. At these frequencies, both double Compton and free-free processes play an important role, as their time scales are $\propto x_{e}^{2}$. Double Compton processes are more important as compared to free-free emission/absorption at higher redshifts as they scale as $(1+z)^{5}$ while free-free processes scale as $(1+z)^{5 / 2}$. For $z<10^{4}$, the free-free absorption is the dominant process (appendix A).

As the efficiency of double Compton and free-free processes is a sharp function of frequency, it partly allows us to isolate the impact of these processes from Compton scattering.

We first consider the case for which double Compton and free-free processes are not efficient. It is further assumed that the brightness temperature of photons, $T_{\mathrm{B}} \gg T_{e}$, or the energy density of injected photons far exceeds the energy density of the equilibrium Planckian

\footnotetext{
${ }^{3}$ The r.h.s. vanishes for only this function. However, if only Compton and inverse Compton scatterings are considered, another equilibrium solution is Bose-Einstein distribution function with non-zero chemical potential.
} 
in the relevant frequency range. For $x_{e} \ll 1$, the equilibrium photon occupation number, $\eta \simeq 1 / x_{e} \gg 1$. With the injection of additional photons, the photon occupation number becomes $\eta \simeq 1 / x_{B}$, with $x_{B}=h \nu /\left(k T_{B}\right)$. As $x_{B} \ll x_{e}$, the $\eta^{2}$ term (the term corresponding to stimulated emission) in eq. (3.1) dominates the other two terms and eq. (3.1) reduces to $([77-80])$ :

$$
\left(\frac{\partial \eta}{\partial y}\right)_{C}=\frac{1}{x_{e}^{2}} \frac{\partial}{\partial x_{e}}\left(x_{e}^{4} \eta^{2}\right)
$$

After substitution: $f\left(x_{e}, y\right)=x_{e} \eta^{2}$, this equation reduces to Burgers equation, which corresponds to the formation of a one-dimensional shock, with solution (see also [6]):

$$
f=g\left(x_{e}+2 y f\right)
$$

where $g(\ldots)$ is any well-behaved function which provides the initial profile of the injected photons. The behaviour of eq. (4.3) can be gauged from the well-known solution to Burgers equation. It corresponds to the excess photon profile moving towards lower frequency with a speed $2 f a_{c}$. The speed is higher for larger value of $f$, just like the passage to a shock solution. This results in a steepening of the initial profile which cause the $d \eta / d x_{e}$ term to become important in eq. (3.1).

In other words, if the injected energy dominates the background intensity in a narrow range of frequencies such that $\eta \gg 1 / x_{e}$ and its profile is smooth, the dominant process that determines the initial evolution of the profile is the Compton scattering, which arises from the recoil of electron in its rest frame. The Compton scattering causes the photons to lose energy to electrons at a rate $f a_{c}$, which depends on both the height of the profile and the Compton energy exchange time scale. However, the validity of eq. (4.2) breaks down after the initial phase because its solution (eq. (4.3)) results in the steepening of the profile. As the profile steepens, the $d \eta / d x_{e}$ term in eq. (3.1) cannot be neglected. As noted above, this term corresponds to energy exchange owing to the motion of electrons in the lab frame (inverse Compton scattering) and its net impact is to heat the photons. When the inverse Compton scattering becomes important, the photons start gaining energy. (This situation is analogous to the solution of Burgers equation for the one-dimensional shock solution. When velocity gradients become large close to the shock front, the viscosity cannot be neglected, resulting in the dissipation of energy and smoothening of the shock profile.)

The effect of these two competing processes is to shift a majority of excess photons to smaller frequencies during the initial phase, followed by the smoothening of the profile which upscatters a fraction of the photons to higher frequencies. ${ }^{4}$ In the inverse Compton scattering, the photons gain energy as $\propto \exp (y)$. As $y \simeq 1$ at $z \simeq 2 \times 10^{5}$ and it scales as $(1+z)^{2}$ (in radiation-dominated era) this gain could be negligible if the energy is injected at a later redshift. If the injection of the energy is at redshifts such that $y<1$, the inverse Compton scattering cannot populate photons at frequencies much larger than the frequency range of injected photons. This idealized case gives us important insights into the numerical solutions of Kompaneets equation and we will compare our numerical solutions with eq. (4.3) in the next section.

\footnotetext{
${ }^{4}$ It is easier to see how the two terms in eq. (3.1) compete if $x_{e}$ is explicitly written in terms of $\nu$ and electron temperature $T_{e}$. In equilibrium $T_{e}=T_{\mathrm{CMB}}$ these terms cancel each other. If $T_{e}>T_{\mathrm{CMB}}$, the inverse Compton scattering term dominates. In our case, $T_{e} \simeq T_{\mathrm{CMB}}$ even after the energy injection as these temperatures are determined by ratio of injected and equilibrium energy density which is very small. Therefore, in our case, the inverse Compton scattering becomes important owing to steepening of the $\eta$ profile. This also allows us to see how equilibrium is attained when only Compton/inverse Compton processes operate.
} 
The absorption and emission of photons owing to double Compton and free-free processes (eqs. (3.2) and (3.4)) also have significant impact on the evolution of the photon occupation number for $x_{e} \ll 1$ in the redshift range of interest, as the time scales for these processes scale as $x_{e}^{2}$ (appendix A). If photons are injected such that $T_{B} \gg T_{e}$ in a small range of frequencies for $x_{e} \ll 1$, eqs. (3.2) and (3.4) show that the dominant process is the absorption of these photons. A fraction of this absorbed energy heats electrons (eq. (3.6)) and another fraction is re-emitted as soft photons. The loss of energy owing to expansion is not important as the time scale of free-free processes, which are the dominant processes for $z<10^{4}$, is shorter than or comparable to the expansion rate before recombination.

We next consider the evolution of the electron temperature (eq. (3.6)). To simplify eq. (3.6), let us write photon occupation number as:

$$
\eta\left(t, x_{e}\right)=\eta_{0}\left(x_{e}\right)+\delta \eta\left(x_{e}, t\right)
$$

Here $\eta_{0}\left(x_{e}\right)$ is the unperturbed distribution function at $T_{\mathrm{CMB}}=T_{e}$. We note that this split is not a perturbation expansion because $\delta \eta$ could dominate the equilibrium distribution for a range of frequency. However, electron temperature is determined by integrated quantities such as photon energy density which are dominated by equilibrium distribution function. Therefore, while such a split would be less appropriate for studying the evolution of photon occupation number, it is suitable for studying the evolution of electron temperature. Using eqs. (3.7) and (3.9) and dropping the logarithmic frequency dependence of $g\left(x_{e}\right)$, we get:

$$
\frac{d T_{e}}{d t}=-\frac{2 \dot{a}}{a} T_{e}+\frac{\left(T_{\mathrm{eq}}-T_{e}\right)}{t_{\mathrm{e} \gamma}}+\frac{T_{e}}{t_{\mathrm{ff}}} \int \delta \eta x_{e} d x_{e}+\frac{T_{e}}{t_{\mathrm{dc}}} \int \delta \eta x_{e} d x_{e}
$$

In writing the free-free and double Compton terms in eq. (4.5) we have assumed that the support of $\delta \eta$ is in small range of frequencies for $x_{e} \ll 1$. We expect the fractional change in the temperature to be small as compared to the unperturbed temperature, which allows us to replace temperature in $x_{e}$ with unperturbed temperature. Eq. (4.5) can then be solved to give:

$$
\begin{aligned}
T_{e}(t) \simeq & T_{e}\left(t_{i}\right) \exp \left[-\int_{t_{i}}^{t} d t^{\prime}\left(\frac{1}{t_{\mathrm{e} \gamma}}+\frac{2 \dot{a}}{a}+\frac{1}{t_{\mathrm{ff}}^{\prime}}+\frac{1}{t_{\mathrm{dc}}^{\prime}}\right)\right]+\exp \left[-\int_{t_{i}}^{t} d t^{\prime}\left(\frac{1}{t_{\mathrm{e} \gamma}}+\frac{2 \dot{a}}{a}+\frac{1}{t_{\mathrm{ff}}^{\prime}}+\frac{1}{t_{\mathrm{dc}}^{\prime}}\right)\right] \\
& \times \int_{t_{i}}^{t} d t^{\prime} \exp \left[-\int_{t_{i}}^{t^{\prime}} d t^{\prime \prime}\left(\frac{1}{t_{\mathrm{e} \gamma}}+\frac{2 \dot{a}}{a}+\frac{1}{t_{\mathrm{ff}}^{\prime}}+\frac{1}{t_{\mathrm{dc}}^{\prime}}\right)\right] \frac{T_{\mathrm{eq}}}{t_{\mathrm{e} \gamma}}
\end{aligned}
$$

Here we have redefined:

$$
\begin{aligned}
\frac{1}{t_{\mathrm{ff}}^{\prime}} & =\frac{1}{t_{\mathrm{ff}}} \int \delta \eta x_{e} d x_{e} \\
\frac{1}{t_{\mathrm{dc}}^{\prime}} & =\frac{1}{t_{\mathrm{dc}}} \int \delta \eta x_{e} d x_{e}
\end{aligned}
$$

For all the cases we consider, the integral over $\delta \eta$ is less than $10^{-6}$. This, in addition with a comparison between different time scales, (eqs. (A.2), (A.4), (A.6), and (A.7)), show that the evolution of the matter temperature is predominantly governed by the inverse Compton scattering time scale. First, this means all terms except the inverse Compton term in eq. (4.6) can be dropped. As the inverse Compton scattering rate far exceeds the expansion rate, the first term on the r.h.s. of the equation can also be dropped. By making a change of variables 
in the second term, we get $T_{e} \simeq T_{\text {eq }}$ (see e.g. [81] for a similar physical setting). $T_{\text {eq }}$ can be computed by solving the evolution of perturbed photon occupation number using Kompaneets equation (eqs. (3.7) and (3.5)). In all the cases we consider, $T_{e}-T_{e 0} \ll T_{e 0}$, where $T_{e 0}$ is the unperturbed temperature.

Using the expansion given by eq. (4.4), we get:

$$
T_{\text {eq }}=\frac{T_{e 0} \int_{0}^{\infty} d x_{e} x_{e}^{4}\left(\eta_{0}\left(\eta_{0}+1\right)+\delta \eta\left(\eta_{0}+1\right)+\delta \eta^{2}\right)}{4 \int_{0}^{\infty} d x_{e} x_{e}^{3}\left(\eta_{0}+\delta \eta\right)}
$$

\section{Numerical solution of Kompaneets equation}

As discussed in the last section, our aim is to study the evolution of the photon occupation number if the energy density of injected photons dominates the equilibrium distribution function, $T_{\mathrm{B}} \gg T_{e}$, for a small range of frequencies with $x_{e} \ll 1$. We discussed possible analytical solutions in such a case taking into account Compton and inverse Compton scattering. We also showed that electron temperature relaxes to an effective radiation temperature given by eq. (4.9), which can be defined for arbitrary radiation fields, on time scales much shorter than all the other time scales (appendix A). This allows us to split the problem of simultaneous determination of photon occupation number and electron temperature into one in which slower processes determine the photon occupation number while the electron temperature varies according to $T_{e}=T_{\mathrm{eq}}$.

In this section, we seek numerical solutions to eq. (3.5). It is a one-dimensional PDE in variables $x_{e}$ and $y$ coupled to the evolution of electron temperature (eq. (3.6)). ${ }^{5}$ We used MATLAB to solve eq. (3.5), making use of the inbuilt PDE solver (pdepe). In order to be able to use the inbuilt solver to solve this equation, we convert it into the following form:

$$
c\left(x, t, u, \frac{\partial u}{\partial x}\right) \frac{\partial u}{\partial t}=x^{-m} \frac{\partial}{\partial x}\left(x^{m} f\left(x, t, u, \frac{\partial u}{\partial x}\right)\right)+s\left(x, t, u, \frac{\partial u}{\partial x}\right)
$$

The variable $\eta x_{e}^{3}$ is used for numerical stability.

We assume two initial profiles for solving the Kompaneets equation: Gaussian and the profile corresponding to a decay product (to be discussed in the next section). The initial photon occupation number is given by $\eta\left(x_{e}, z_{i}\right)=\delta \eta\left(x_{e}, t_{i}\right)+\eta_{0}\left(x_{e}\right)$, where $\eta_{0}\left(x_{e}\right)$ is the equilibrium distribution and $\delta \eta$ is the profile of the injected energy.

We first consider the Gaussian profile, which is given by:

$$
x_{e}^{3} \delta \eta\left(x_{e}, z_{i}\right)=\frac{A}{\sigma \sqrt{2 \pi}} \exp \left[-\left(x_{e}-\mu\right)^{2} /\left(2 \sigma^{2}\right)\right]
$$

Here $z_{i}$ is the redshift at which the energy is injected. $A$ is an overall normalization that determines the total amount of injected energy. In addition to $t_{i}$ and $A$, the mean and the standard deviation of the Gaussian, $\mu$ and $\sigma$ give the other two free parameters for our study.

We fix $\mu \simeq 2 \times 10^{-3}$ as required by the EDGES detection. For $z_{i} \gg 10^{5}, y \gg 1$, which means Compton scattering time scales are short enough to thermalize the excess of photons. This motivate us to consider $z_{i}<10^{5}$ for our study. The range of $A$ is fixed by

\footnotetext{
${ }^{5}$ We note that the use of variable $x_{e}$ could be misleading when these two equations are coupled as electron temperature enters the definition of $x_{e}$. We use the variable $x_{e}$ with unperturbed electron temperature in this case and redefine $x_{e}$ as $x_{e}=h \nu /\left(k T_{e 0}\right)$ and use the electron temperature explicitly in eq. (3.5). However as $T_{e}-T_{e 0} \ll T_{e 0}$, our solutions are insensitive to the use of either of the two variables.
} 

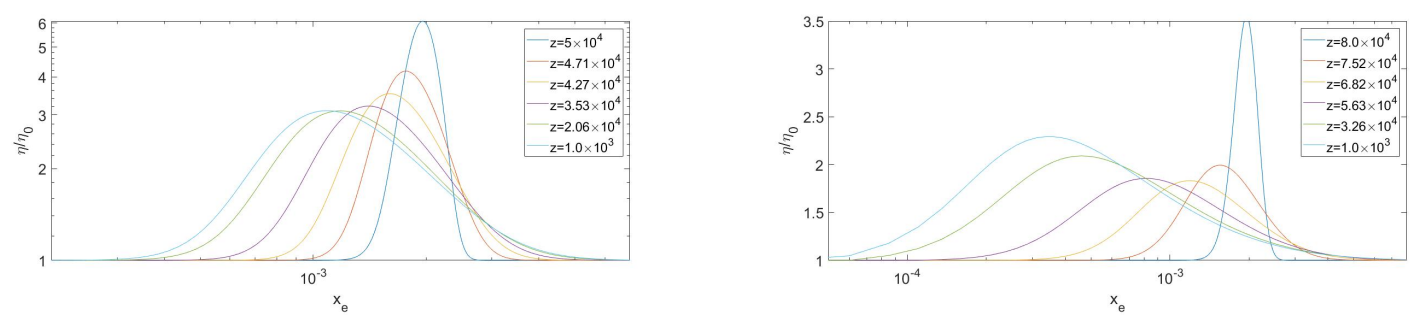

Figure 1. The figures display the solution of eq. (3.1) for models in which a Gaussian profile is injected with mean $x_{e}=2 \times 10^{-3}$ at two different redshifts. The y-axis displays the ratio of the perturbed and the equilibrium CMB occupation number while the x-axis is in units $x_{e} \equiv h \nu /\left(k T_{e 0}\right)$.

the EDGES observation. For $x_{e} \ll 1, \eta \simeq 1 / x_{e}$ for a Planckian. $A$ is chosen such that the brightness temperature of injected photons, $T_{B} \gg T_{e}$, as the EDGES detection requires the excess of photons to be at least a factor of 2.5 more than the unperturbed CMB spectrum at $x_{e} \simeq 2 \times 10^{-3}$.

To gauge the importance of different physical processes, we show results for two different cases: with only Compton/inverse Compton scattering and including all the physical effects (Compton, double Compton and free-free processes). In figure 1, we show numerical solutions to eq. (3.5) for only Compton processes. The evolution of the profile is displayed for a range of redshifts with $z<8 \times 10^{4}$. The profile stops evolving at $z \simeq 1000$ as the universe recombines which causes all the relevant time scales to become much larger than the expansion rate.

Figure 1 shows that the numerical results agree with the analytic solutions (eq. (4.3)): the Gaussian profile moves to lower energies with the steepening of the profile as the dominant process corresponds to stimulated emission $\left(\eta^{2}\right.$ term in eq. (3.1)). As the profile evolves and/or the injection energy is pumped at an earlier era, the $\partial \eta / \partial x$ (inverse Compton scattering) starts playing a role, leading to up-scattering of the low energy photons. As $y<1$ and the excess photons upscatter to frequencies $x_{f} \simeq x_{i} \exp (y)$, the inverse Compton scattering creates a tail above the frequencies of injection in the profile, as is seen in figure 1 . The net impact of both these effects is to broaden the Gaussian, keeping invariant the number density of excess photons $\propto \eta x_{e}^{3}$ while losing energy $\left(\propto \eta x_{e}^{4}\right)$ through Compton scattering to electrons.

In figure 2 we include all the processes. For a photon excess, double Compton/free-free processes mainly act as sink of photons. This is clearly seen when figures 2 and 1 are compared. With only Compton processes the initial distribution moves to smaller frequencies with a small tail at frequencies larger than injected frequencies owing to inverse Compton scattering. However, most of these photons are absorbed by free-free absorption which dominates at $z<10^{4}$. It should also be noted from figure 2 that free-free absorption continues to deplete photons until $z \simeq 1000$ as its time scale at $x \simeq 10^{-3}$ remains comparable to the expansion time scale until recombination. This also means that the EDGES result, which requires a minimum residual excess of 2.5 at $x_{e} \simeq 2 \times 10^{-3}$, can generically be achieved in the pre-recombination era only if the amount of initial injection far exceeds the minimum required residual excess, as is clear from figure 2.

Figure 2 shows that to explain the EDGES detection the energy injection redshift $z_{i}<$ $4 \times 10^{3}$. For energy injection at higher redshifts, free-free processes equilibrates the surplus photons. It could be examined whether a larger amount of injection could potentially leave a residue. We find this conclusion to be insensitive to the amount of injected energy because this also increases the rate of absorption (eq. (3.4) and (3.2)). 

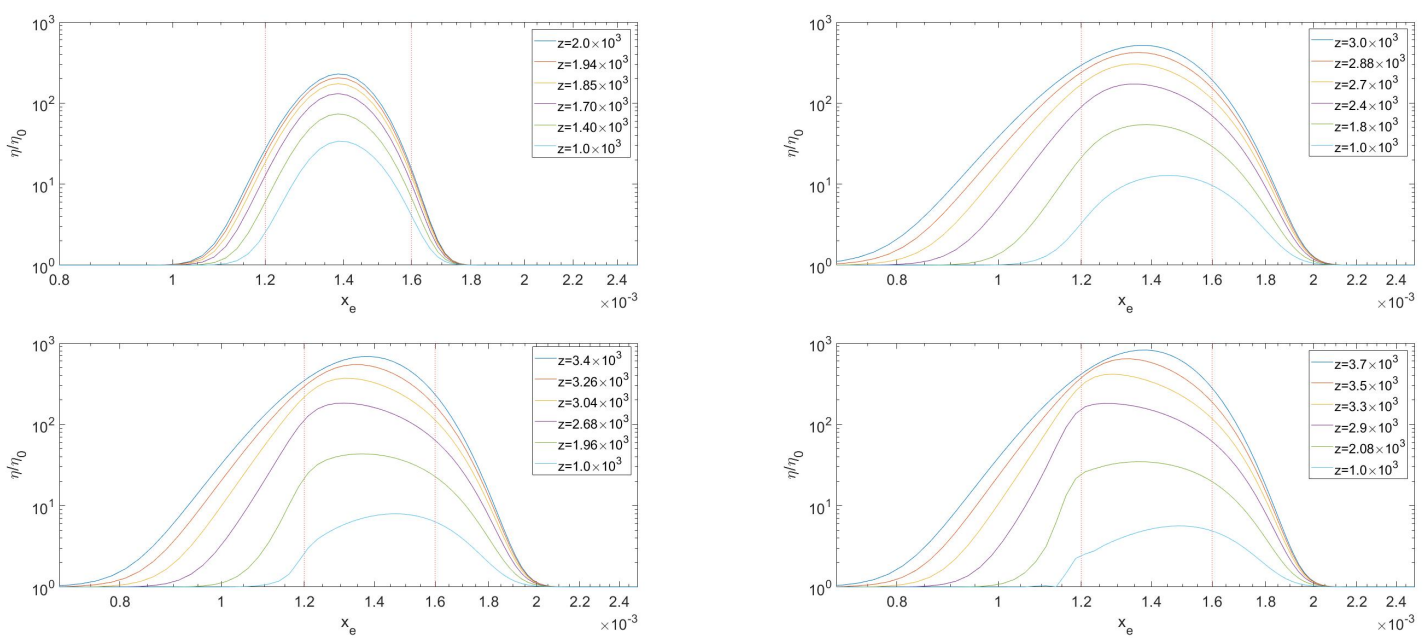

Figure 2. For Gaussian input profiles of different amplitude, width, and starting redshift, the panels show the evolution of the profile, including all the relevant physical processes (eq. (3.5)). The axes display the same quantities as in figure 1 . The vertical lines correspond to $x_{e}=\{1.2,1.6\} \times 10^{-3}$. To explain the EDGES detection, the ratio displayed on y-axis should exceed 2.5 in this range of $x_{e}$.

\subsection{Decaying particle}

In this section, we consider particle decay as the mechanism of pumping photons in the CMB at $x_{e} \ll 1$. In this model, a non-relativistic particle of mass $m_{\mathrm{d}}$ decays into a neutral, massless particle and a photon. To get the photon injection in the desired frequency range, we need to assume that the mass of the particle is such that $m_{\mathrm{d}} c^{2} \ll k T_{e}$. If this particle was coupled to the thermal bath of the universe in the early universe, it would be highly relativistic. Therefore, our implicit assumption is that this particle was always decoupled from the thermal bath (e.g. an axion). For our work we require $m_{\mathrm{d}} \gtrsim 3 \times 10^{-3} \mathrm{eV}$. The occupation number of decay photons could be written as, in radiation dominated era (for details see [81]):

$$
\eta_{\text {decay }}(\nu, t)=\frac{h^{2} B n_{\mathrm{d}}(\tau)}{2 \pi\left(m_{\mathrm{d}} / 2\right)^{2} \nu c}\left(\frac{\tau}{t}\right)^{1 / 2} \exp \left(-\frac{t}{\tau}\left(\frac{2 h \nu}{m_{\mathrm{d}} c^{2}}\right)^{2}\right) \Theta\left(\frac{m_{\mathrm{d}} c^{2}}{2}-h \nu\right)
$$

Here $B$ is the branching ratio of photon decay. The initial conditions are set in the radiation dominated era; we make the switch to the relevant expression in the matter dominated era at matter radiation equality (for details see [81]). We can compute the ratio of the photon occupation number of decay photons and CMB photons for $x_{e} \ll 1$. Using $n_{\mathrm{d}}(0) m_{\mathrm{d}} / \rho_{c}(0)=$ $\Omega_{\mathrm{d}}$ and, for CMB for $x_{e} \ll 1, \eta_{0}=1 / x_{e}$, we get, in the radiation dominated era:

$$
\frac{\eta_{\text {decay }}(\nu, t)}{\eta_{0}}=\frac{2 B h^{3} \rho_{c}(0) \Omega_{\mathrm{d}}\left(1+z_{d}\right)^{3}}{\pi m_{\mathrm{d}}^{3} c\left(k T_{e}\right)}\left(\frac{\tau}{t}\right)^{1 / 2} \exp \left(-\frac{t}{\tau}\left(\frac{2 h \nu}{m_{\mathrm{d}} c^{2}}\right)^{2}\right) \Theta\left(\frac{m_{\mathrm{d}} c^{2}}{2}-h \nu\right)
$$

Here $z_{d}$ is the redshift corresponding to $t=\tau$. It should be noted that $\eta_{\text {decay }}(\nu, t) / \eta_{0}$ is independent of both redshift and frequency for $t \ll \tau$.

It is easy to verify that our requirement, $\eta_{\text {decay }}(\nu, t) / \eta \gg 1$, can readily be achieved for a wide range of $m_{\mathrm{d}}$ and $\tau$ for $\Omega_{\mathrm{d}} \ll 1$. 

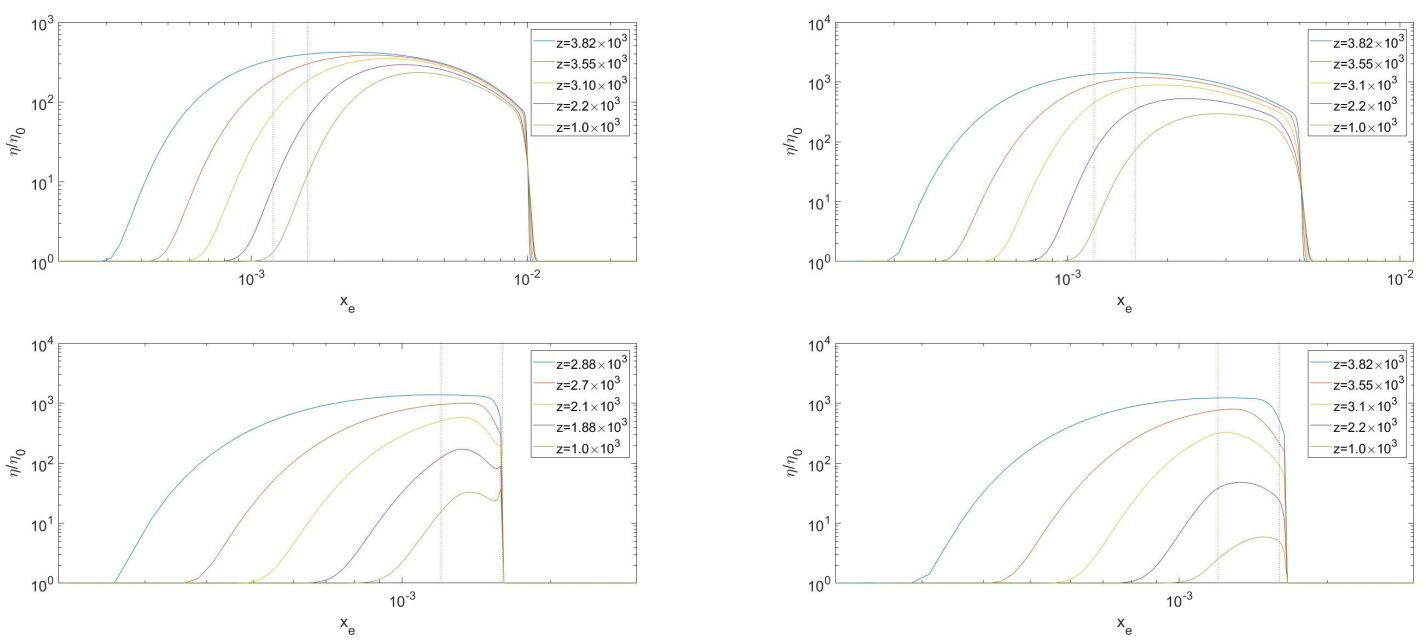

Figure 3. The evolution of the photon occupation number is shown for decaying particle model (eq. (5.4)) for different values of decay redshift, $z_{d}$, and particle mass, $m_{d}$. The panels (clockwise from the top left) correspond to $z_{d}=\left\{5.6 \times 10^{3}, 5.6 \times 10^{3}, 2 \times 10^{3}, 3 \times 10^{3}\right\}$ and $m_{d}=\left\{2 \times 10^{-2}, 9 \times\right.$ $\left.10^{-3}, 3.1 \times 10^{-3}, 3 \times 10^{-3}\right\} \mathrm{eV}$, respectively. The axes display the same quantities as in figure 2 .

In figure 3, the evolution of an input energy profile given by eq. (5.4) is shown for different decay times $\tau$ and particle mass $m_{d}$.

To understand the figure and its implication, the following two facts must be noted. First, all photons have the frequency, $\nu=m_{d} c^{2} / h$, at the time of production. These photons subsequently free stream in the expanding universe, losing their energy owing to redshift. Therefore, at any fixed time, the photons with frequency $\nu \ll m_{d} c^{2} /(2 h)$ are old photons that are absorbed with a greater probability because the rate of absorption is greater at higher redshifts. Second, the photon production is a continuous process. For $t \ll \tau$, the number of decay photons, $\simeq n_{d} t / \tau \ll n_{d}$. A majority of these photons are absorbed and thermalized. While these photons do not play a role in explaining the residual, they are responsible for spectral distortion of the CMB which will be discussed in the next section. The photons that contribute to the observed excess are produced at $t \simeq \tau$. Therefore, to explain the residual photons at $z \simeq 1000$, we only need to model the late time evolution of the decay photons and it suffices to evolve photons close to the energy $m_{d} c^{2} / 2$ at times close to $\tau$. We choose $z_{i}=z_{d}$ as our initial condition. We note that our results are insensitive to the choice of $z_{i}$ so long as $z_{i} \gtrsim z_{d}$ and we verify this by running our code with different $z_{i}$ up to $z_{i} / z_{d}=10$.

\subsection{Explaining EDGES data}

In section 2, we discussed the EDGES result and different ways to interpret it. One possible explanation is to increase $T_{\mathrm{CMB}}$ in eq. (2.2) by at least a factor of 2.5 in the observed frequency range $70-90 \mathrm{MHz}$, which corresponds to $x_{e}=\{1.2,1.6\} \times 10^{-3}$. The range is marked in figures 2 and 3.

In the Gaussian model, the requisite ratio of the residual excess $T_{B} / T_{e}$ can be achieved by varying $z_{i}$ and $A$. As noted above, if the redshift of injection $z_{i}>5 \times 10^{3}$, the freefree absorption causes the injected feature to be thermalized. For $z_{i}<4 \times 10^{3}$ we can get the desired ratio for the input Gaussian profile (figure 2). Figure 2 further shows that $T_{B} / T_{e} \gtrsim 200$ for acceptable models. 
For the decaying particle model, we find that $m_{d} \simeq 3 \times 10^{-3} \mathrm{eV}$ and $z_{d}<4 \times 10^{3}$ to explain the EDGES data (figure 3). We note that the EDGES detection constrains both $z_{d}$ and $m_{d}$.

All the cases shown in figure 2 and three of the four cases shown in figure 3 can explain the EDGES data.

\section{CMB spectral distortion}

In this paper, we invoke spectral distortion of $\mathrm{CMB}$ at $x_{e} \simeq 10^{-3}$ to explain the EDGES result. In addition to these frequencies, physical processes in the pre-recombination era also impact other parts of the CMB spectrum, which we term 'global' and 'local' spectral distortion.

The energy injected into the plasma heats electrons which, through inverse Compton scattering, transfer a part of their energy to the CMB. ${ }^{6}$ This results in 'global' spectral distortion as the energy can be transferred to CMB photons of any frequency. The other 'local' spectral distortion is caused by the upscattering/reemission of injected photons close to the frequency of injected photons.

global spectral distortion: The global CMB distortion is a two stage process: transfer of a fraction of injected photon energy (at $x_{e} \simeq 10^{-3}$ ) to electrons followed by a transfer of a fraction of this energy to CMB at other frequencies (e.g. [81] for details). The dominant physical process for the former is free-free absorption and inverse Compton scattering for the latter. Eqs. (A.2) and (A.3) show that the time scales of these processes are shorter than the expansion time in the pre-recombination era. This means that the loss of energy due to the expansion of the universe during the energy exchange is insignificant. As $T_{e} \simeq T_{\text {eq }}$, the thermal energy density of baryons $\simeq n_{e} k T$ is negligible as compared to the CMB energy density, which implies a negligible amount of injected energy is retained by particles.

Therefore, it is reasonable to assume that, apart from the residual, unthermalized energy, all the injected energy is transferred to CMB. Figures 2 and 3 show that the residual is generally a small fraction of the total injected energy. Using eq. (4.1) we can estimate the amount of energy absorbed by the CMB. We require $\eta / \eta_{0} \simeq 2.5$, or $T_{\mathrm{B}} / T \simeq 2.5 \mathrm{in}$ eq. (4.1), to explain the EDGES result. However, figure 2 shows that a much larger amount of energy, $\eta / \eta_{0} \simeq 200-1000$, has to be injected to achieve the requisite residual. This yields $\Delta \rho_{\mathrm{CMB}} / \rho_{\mathrm{CMB} 0} \simeq 10^{-7}-10^{-6}$, and it is a generic prediction of the model.

The case of decaying particles is more complicated as the injection is a continuous process. To capture the time dependence of the energy exchange, we assume that all the decay photons are absorbed at the redshift of production. This is a reasonable assumption for photons close to $x_{e} \simeq 10^{-3}$ as seen in figure 3 . The energy density of the decay photons is $\rho_{\mathrm{d} \gamma}(z) \simeq m_{\mathrm{d}} c^{2} n_{d}(z)(1-\exp (-t / \tau)) / 2 ; n_{\mathrm{d}}(z)=n(0)(1+z)^{3} \exp (-t / \tau)$ is the number density of decay particles. At $t \ll \tau, \rho_{\mathrm{d} \gamma}(z)$ scales as $(1+z)$ and, even though the transfer of energy from decay photons to CMB is more efficient, the ratio of decay photons and CMB energy density scales $(1+z)^{-3}$. This means the fractional change in CMB energy is smaller at higher redshifts. The main contribution to CMB distortion arises from $z \lesssim z_{d}$, which allows us to compute the spectral distortion as in the previous case. The EDGES detection requires $z_{d} \lesssim 4 \times 10^{3}$ and $\eta_{\text {decay }} / \eta_{0} \simeq 100-1000$, which corresponds to $\Omega_{\mathrm{d}} \simeq 10^{-6}-10^{-5}$

\footnotetext{
${ }^{6}$ Our main focus is $y$-distortion which is caused if the energy is injected at $z \lesssim 10^{5}$. In this case, the rate of Compton processes is smaller than the expansion rate (eq. (A.1)), which prevents relaxation to an equilibrium distribution (e.g. $[18,81]$ and references therein).
} 
for $B=1$ (eq. (5.4) and figure 3). This yields fractional change in CMB energy density: $\Delta \rho_{\mathrm{CMB}} / \rho_{\mathrm{CMB} 0} \simeq 10^{-6}-10^{-5}$.

The COBE-FIRAS data yields the best upper limit on the CMB spectral distortion: $\Delta \rho_{\mathrm{CMB}} / \rho_{\mathrm{CMB} 0}<6 \times 10^{-5}$ for $\nu \gtrsim 60 \mathrm{GHz}$. Our predicted spectral distortion does not violate the COBE-FIRAS bounds, and its detection is within the capabilities of the upcoming satellite mission PIXIE ([23]).

local spectral distortion: The 'local' CMB distortion corresponds to the redistribution of injected photons higher up the frequency ladder close to the frequency of injection. This is of particular interest because CMB spectrum has been determined to high precision upto $\nu \simeq 1.5 \mathrm{GHz}$, which is only roughly a factor of 20 more than the energy of injection [82]. ${ }^{7}$ The energy injection in the early universe can be constrained by ARCADE results [83]. Figures 3 and 2 show we do not expect significant emission at frequencies much larger than the injection frequencies. We can explain it by considering the relevant physical processes.

Three physical processes can cause an increase in the frequency, through re-emission or scattering, of injected photons: inverse Compton scattering, free-free emission, and double Compton emission. As the time scale of free-free/double Compton processes scales as $x_{e}^{2}$, they are generally inefficient at re-emitting photons at frequencies 10 times larger than the energy of injection. ${ }^{8}$ So the dominant process that re-distributes energy to higher frequency is inverse Compton scattering, as our numerical results show. As the rate of inverse Compton scattering is smaller than the free-free absorption and the process raises the frequency as $\exp (y)$, we do not expect significant excess at frequencies separated from the frequency of injection so long as $y \leq 1$.

This means the 'local' spectral distortion could be negligible even if a large amount of energy is injected. Alternatively, we could seek to explain, or put constraints from, ARCADE results by adjusting the time of injection of energy and for the decaying particle model, $m_{d}$. One of the panels of figure 3 (top left) shows a model in which ARCADE result at $408 \mathrm{MHz}$ is violated. However, this model is not consistent with the EDGES results for the following reason: for causing substantial distortion at frequencies probed by ARCADE result, $m_{d}$ needs to be much larger than the value required to explain the EDGES result. This means photons close to $x_{e} \simeq 1.5 \times 10^{-3}$ are old photons, as explained above, and are absorbed more efficiently at higher redshifts.

\subsection{Impact on CMB anisotropies}

The injection of photons at $x_{e} \ll 1$ could leave detectable imprints on CMB anisotropies for the following reasons:

1. The change in the matter/radiation content of the universe alters the matter-radiation equality.

2. The photons and baryons are tightly coupled before the recombination commences, as the time scale of photon-electron scattering, $t_{c}=1 /\left(n_{e} \sigma_{T} c\right)$, is shorter than the

\footnotetext{
${ }^{7}$ We refer to frequencies $\nu=\{3.2,1.42\} \mathrm{GHz}$ of ARCADE data here at which the extragalactic residual is $2.787 \pm 0.01 \mathrm{~K}, 3.18 \pm 0.516 \mathrm{~K}$, respectively. We could also consider the data point at $\nu=408 \mathrm{MHz}$ at which the extragalactic residual is $10.8 \pm 3.5 \mathrm{~K}$.

${ }^{8}$ Even if the time scale of emission is shorter than expansion time scale, the absorption time scales are shorter than emission time scales if there is a photon excess (eqs. (3.4) and (3.2)). The net effect of these processes is to create an equilibrium photon occupation number of temperature $T_{e}$ at frequencies smaller than the $x_{e}$ at which these time scales are shorter than expansion time scale.
} 
expansion time scale during this era. The extra radiation energy, tightly coupled to baryons, results in lower baryon loading $R \equiv 3 \rho_{b} /\left(4 \rho_{\gamma}\right)$, which is strongly constrained by CMB anisotropies (for details see e.g. [84] and references therein).

The impact of these effects can be studied within the framework of a specific model, e.g. decaying particle scenario we outlined above. In this model, a non-relativistic particle of density parameter $\Omega_{\mathrm{d}}$ contributes to the cold dark matter of the universe before it decays into radiation on a time scale $\tau$. The relative impact of this model on CMB anisotropies can be discerned from the range of permissible values of $B$ and $\Omega_{\mathrm{d}}$ from Planck CMB data.

From eq. (5.4) it follows that, for $B=1$, the ratio of decay photons to CMB occupation number is unity for $\Omega_{\mathrm{d}} \simeq 10^{-8} \Omega_{m}$ (for $z_{d}=3 \times 10^{3}$ ), where $\Omega_{m}=0.315 \pm 0.007$ is the best-fit matter density parameter from Planck. To explain the EDGES result, we require $\Omega_{\mathrm{d}} \simeq 10^{-5}$ which is well within the precision with which $\Omega_{m}$ has been determined by Planck. However, for $B \ll 1$, the case for which only a small fraction of particles decay into photons, $\Omega_{\mathrm{d}}$ needs to be larger by a factor $1 / B$ to explain the EDGES result. It should be noted that the amplitude of CMB spectral distortion also depend on the product, $B \Omega_{\mathrm{d}}$. However, CMB anisotropies are sensitive to $\Omega_{\mathrm{d}}$ in addition. Therefore, CMB anisotropies provide a complementary probe of the decaying particle model.

Planck data measures the angular acoustic scale with $0.03 \%$ precision [1]. The angular acoustic scale is $\propto 1 /(1+R)^{1 / 2}$. From eq. (4.1) it follows that the additional energy $\delta \rho_{\gamma} \simeq$ $10^{-9} \rho_{\mathrm{CMB}}$ for $T_{B}=T_{e}$. For explaining the EDGES result we require $T_{B} / T_{e} \lesssim 1000$ which causes a fractional change $\Delta R / R \simeq 10^{-6}$, which is well within Planck constraints. Similar conclusions can be reached for the decaying particle model.

Therefore, the scenario studied in this paper is consistent with Planck data. However, decaying particle models with $B \ll 1$ might yield observable features in CMB data and we hope to return to this study in the future.

\section{$7 \quad$ Summary and conclusions}

In this paper we study the possibility of explaining the recent EDGES detection using energy injection in CMB at $x_{e} \ll 1$ during the pre-recombination era. We study, both analytically and numerically, the evolution of the CMB spectrum in the presence of energy injection of arbitrary amplitude and redshift of injection. All the important physical processes Compton/inverse Compton scattering, free-free absorption/emission, and double Compton absorption/emission - are considered in our study. We analyze two different models of energy injection: (a) Gaussian profile with varying amplitude and injection redshifts, (b) decay of a non-relativistic particle parameterized in terms of its number density, mass, and decay redshift.

We show that if the energy injection in the relevant frequency range $\left(x_{e} \simeq 2 \times 10^{-3}\right)$ occurs after $z \simeq 4 \times 10^{3}$, the energy density of the residual, unthermalized photons in the CMB can explain the EDGES detection. Figures 3 and 2 also show that, for a generic injection event, the injected energy needs to be more than two orders of magnitude larger than the minimum residue required to explain the EDGES detection.

We find that the energy injection would also cause: (a) 'global' y-distortion of CMB owing to the heating of electrons, (b) 'local' distortion of the CMB owing to the upscattering of injected photons, (c) additional CMB anistropies because of the increased matter and radiation energy density. 
The 'global' y-parameter is currently constrained by COBE-FIRAS observation to be $\lesssim 10^{-5}$. The upcoming instrument PIXIE will improve this by up to four orders of magnitude. The 'local' distortion is constrained by ARCADE observations. Planck results have precisely determined both the radiation and matter energy density in the universe. We show that our proposed scenario satisfies all these constraints. Therefore, the model we consider is tightly constrained by current observations and make meaningful predictions for the future observations. In particular, PIXIE might be able to detect such an energy injection in the pre-recombination era.

It might be possible to distinguish the energy injection in the pre-recombination era from other mechanisms that have been invoked to explain the EDGES detection in the postrecombination era, e.g. production of photons owing to radio sources or the milli-charged dark matter particle. Figures 3 and 2 show that there are no residual photons for $x_{e}<10^{-3}$. This is a generic feature of the pre-recombination physical processes as the free-free absorption time scale for these photons is shorter than the expansion time scale even close to the epoch of recombination. However, the low-redshift radio background is unlikely to have such a cut-off and, therefore, it will also impact the pre-reionization EoR absorption feature at $\nu \simeq 30 \mathrm{MHz}$. The proposed mission FARSIDE, which is capable of detecting the pre-reionization HI signal, will be able to distinguish between these models. ${ }^{9}$

While the main aim of this paper is to explain the EDGES detection, we have presented a general formalism to study the evolution of energy injection in the pre-recombination era at $x_{e} \ll 1$. It allows for the injected energy to far exceed the CMB energy for a small range of frequencies if the total amount of injected energy remains small as compared to the total CMB energy.

\section{A Time scales of various radiative processes}

In this appendix, we list different time scales relevant for our study.

Compton/inverse Compton scattering. For Compton/inverse Compton scattering, an important time scale is the energy relaxation time scale between electrons and photons if electron is the target. This time scale determines the evolution of the photon occupation number:

$$
t_{\gamma e}=\left(\frac{1}{n_{e}(z) c \sigma_{\mathrm{T}}}\right)\left(\frac{m_{e} c^{2}}{k T_{\mathrm{CMB}}}\right)=4.8 \times 10^{13}\left(\frac{10^{4}}{1+z}\right)^{4} \mathrm{sec}
$$

Similarly, we can define a relaxation time scale if photon is the target. This time scale determines the evolution of matter temperature.

$$
t_{e \gamma}=\frac{3 m_{e} c}{4 \sigma_{\mathrm{T}} \rho_{\mathrm{CMB}}}=7.3 \times 10^{3}\left(\frac{10^{4}}{1+z}\right)^{4} \mathrm{sec}
$$

Free-free emission/absorption. The relevant time scale for the evolution of photon occupation number is $x_{e}^{3} \eta /\left(g\left(x_{e}\right) K_{0}\right)$ (eq. (3.4)) with ${ }^{10}$

$$
K_{0}^{-1}(z)=\left(\frac{8 \pi^{1 / 2} e^{6} h^{2} n_{e}^{2}(z)}{(54)^{1 / 2} m_{e}^{3 / 2}\left(k T_{\mathrm{CMB}}\right)^{7 / 2}}\right)^{-1}=5.3 \times 10^{16}\left(\frac{10^{4}}{1+z}\right)^{5 / 2} \mathrm{sec}
$$

\footnotetext{
${ }^{9}$ https://www.lpi.usra.edu/leag/white-papers-astronomy/FARSIDE_190710_Final.pdf.

${ }^{10}$ see e.g. [6]; $K_{0}$ is more readily derived by starting with the emissivity of thermal free-free emission (e.g. [63]) and diving by $c^{3} /\left(8 \pi h \nu^{3}\right)$ to convert from emissivity to the time derivative of the photon occupation number; this yields $K_{0} / x_{e}^{3}$.
} 
For determining the electron temperature, the time scale of interest is (eq. (3.9)):

$$
t_{\mathrm{ff}}=\left(\frac{8 \pi\left(k T_{\mathrm{CMB}}\right)^{4} K_{0}}{c^{3} h^{3} n_{e}(z) k T_{\mathrm{CMB}}}\right)^{-1}=7 \times 10^{7}\left(\frac{10^{4}}{1+z}\right)^{5 / 2} \mathrm{sec}
$$

Double Compton emission/absorption. The relevant time scale for the evolution of the photon occupation number is $x_{e}^{3} \eta / C(t)$ (eq. (3.2)) with

$$
C^{-1}(z)=3.2 \times 10^{19}\left(\frac{10^{4}}{1+z}\right)^{5} \mathrm{sec}
$$

For determining the electron temperature, the time scale is (eq. (3.9)):

$$
t_{\mathrm{dc}}=\left(\frac{8 \pi\left(k T_{\mathrm{CMB}}\right)^{4} C(t)}{c^{3} h^{3} n_{e}(z) k T_{\mathrm{CMB}}}\right)^{-1}=1.1 \times 10^{12}\left(\frac{10^{4}}{1+z}\right)^{5} \mathrm{sec}
$$

Expansion time scale. It is given by the inverse of the expansion rate, $H^{-1}(z)$ :

$$
t_{\exp }=\frac{H_{0}^{-1}}{\left(\Omega_{m}(1+z)^{3}+\Omega_{r}(1+z)^{4}\right)^{1 / 2}} \simeq 4.6 \times 10^{11}\left(\frac{10^{4}}{1+z}\right)^{2} \mathrm{sec}
$$

The numerical value quoted in eq. (A.7) correspond to radiation-dominated era, using $\Omega_{r}=$ $4.3 \times 10^{-5}$.

Finally, we often use the Compton $y$ parameter as the dimensionless time variable. It is given by:

$$
y(t)=\int_{t_{i}}^{t} d t^{\prime} a_{c}
$$

where $t_{i}$ is the time of energy injection and $a_{c}^{-1}$ is the characteristic time scale on which the radiation spectrum reaches a state of quasi-equilibrium under Compton/inverse Compton scattering alone. A useful expression for numerical computation is:

$$
\frac{d y}{d z}=-8.66 \times 10^{-11} \frac{(1+z)^{3 / 2}}{\sqrt{5725+(1+z)}}
$$

The time scales discussed above could change substantially when the departure from the equilibrium state is large. In this paper we study a scenario in which the injected number of photons far exceeds the number of CMB photons in equilibrium for $x_{e} \ll 1$. This means the time scales for the evolution of photon occupation number for $x_{e} \ll 1$ could depend on the number of injected photons. If we assume the brightness temperature in a small frequency range is $T_{B}$ such that $T_{B} \gg T_{e}$, it follows from eqs. (3.2) and (3.4) that the relevant times scales for the evolution of the photon occupation number are $\simeq x_{e}^{3} \eta_{0} T_{e} /\left(C(t) T_{B}\right)$ and $\simeq x_{e}^{3} \eta_{0} T_{e} /\left(K_{0} T_{B}\right)$ for double Compton and free-free processes, respectively. Similarly, as we see in section 4 , the time scale at which photons lose energy to electron through Compton scattering is inversely proportional to the amount of injected energy (eq. (4.3)). However, the times scales of the evolution of electron temperature, determined by eqs. (A.6) and (A.4), are representative as the total amount of energy injected is small as compared to the total CMB energy density. 


\section{References}

[1] Planck collaboration, Planck 2018 results. VI. Cosmological parameters, Astron. Astrophys. 641 (2020) A6 [arXiv:1807.06209] [INSPIRE].

[2] Planck collaboration, Planck 2015 results. XIII. Cosmological parameters, Astron. Astrophys. 594 (2016) A13 [arXiv: 1502.01589] [INSPIRE].

[3] WMAP collaboration, Nine-Year Wilkinson Microwave Anisotropy Probe (WMAP) Observations: Cosmological Parameter Results, Astrophys. J. Suppl. 208 (2013) 19 [arXiv: 1212.5226] [INSPIRE].

[4] BOSS collaboration, The clustering of galaxies in the completed SDSS-III Baryon Oscillation Spectroscopic Survey: baryon acoustic oscillations in the Fourier space, Mon. Not. Roy. Astron. Soc. 464 (2017) 3409 [arXiv: 1607.03149] [InSPIRE].

[5] D.J. Fixsen, E.S. Cheng, J.M. Gales, J.C. Mather, R.A. Shafer and E.L. Wright, The Cosmic Microwave Background spectrum from the full COBE FIRAS data set, Astrophys. J. 473 (1996) 576 [astro-ph/9605054] [INSPIRE].

[6] L. Danese and G. de Zotti, The relic radiation spectrum and the thermal history of the Universe., Nuovo Cim. 7 (1977) 277.

[7] R.A. Daly, Spectral distortions of the microwave background radiation resulting from the damping of pressure waves, Astrophys. J. 371 (1991) 14.

[8] C. Burigana, L. Danese and G. de Zotti, Formation and evolution of early distortions of the microwave background spectrum - A numerical study, Astron. Astrophys. 246 (1991) 49.

[9] W. Hu, D. Scott and J. Silk, Power spectrum constraints from spectral distortions in the cosmic microwave background, Astrophys. J. Lett. 430 (1994) L5 [astro-ph/9402045] [INSPIRE].

[10] R. Khatri, R.A. Sunyaev and J. Chluba, Does Bose-Einstein condensation of CMB photons cancel $\mu$ distortions created by dissipation of sound waves in the early Universe?, Astron. Astrophys. 540 (2012) A124 [arXiv:1110.0475] [INSPIRE].

[11] J. Chluba and R.A. Sunyaev, The evolution of CMB spectral distortions in the early Universe, Mon. Not. Roy. Astron. Soc. 419 (2012) 1294 [arXiv:1109.6552] [InSPIRE].

[12] J. Chluba, R. Khatri and R.A. Sunyaev, CMB at 2x2 order: The dissipation of primordial acoustic waves and the observable part of the associated energy release, Mon. Not. Roy. Astron. Soc. 425 (2012) 1129 [arXiv: 1202.0057] [INSPIRE].

[13] R. Khatri and R.A. Sunyaev, Beyond $y$ and $\mu$ : the shape of the CMB spectral distortions in the intermediate epoch, $1.5 \times 10^{4}<z<2 \times 10^{5}$, JCAP 09 (2012) 016 [arXiv:1207.6654] [INSPIRE].

[14] R. Khatri and R.A. Sunyaev, Creation of the CMB spectrum: precise analytic solutions for the blackbody photosphere, JCAP 06 (2012) 038 [arXiv: 1203.2601] [INSPIRE].

[15] J.B. Dent, D.A. Easson and H. Tashiro, Cosmological constraints from CMB distortion, Phys. Rev. D 86 (2012) 023514 [arXiv: 1202.6066] [INSPIRE].

[16] J. Chluba, A.L. Erickcek and I. Ben-Dayan, Probing the inflaton: Small-scale power spectrum constraints from measurements of the CMB energy spectrum, Astrophys. J. 758 (2012) 76 [arXiv: 1203.2681] [INSPIRE].

[17] J. Chluba, Distinguishing different scenarios of early energy release with spectral distortions of the cosmic microwave background, Mon. Not. Roy. Astron. Soc. 436 (2013) 2232 [arXiv: 1304.6121] [INSPIRE].

[18] H. Tashiro, CMB spectral distortions and energy release in the early universe, PTEP 2014 (2014) 06B107 [INSPIRE]. 
[19] J.C. Hill, N. Battaglia, J. Chluba, S. Ferraro, E. Schaan and D.N. Spergel, Taking the Universe's Temperature with Spectral Distortions of the Cosmic Microwave Background, Phys. Rev. Lett. 115 (2015) 261301 [arXiv:1507.01583] [INSPIRE].

[20] J. Chluba et al., New Horizons in Cosmology with Spectral Distortions of the Cosmic Microwave Background, arXiv: 1909.01593 [INSPIRE].

[21] A. Kogut et al., CMB Spectral Distortions: Status and Prospects, arXiv:1907.13195 [INSPIRE].

[22] J. Chluba, A. Ravenni and S.K. Acharya, Thermalization of large energy release in the early Universe, Mon. Not. Roy. Astron. Soc. 498 (2020) 959 [arXiv:2005.11325] [InSPIRE].

[23] A. Kogut et al., The Primordial Inflation Explorer (PIXIE): A Nulling Polarimeter for Cosmic Microwave Background Observations, JCAP 07 (2011) 025 [arXiv:1105.2044] [INSPIRE].

[24] R. Barkana and A. Loeb, In the beginning: The first sources of light and the reionization of the Universe, Phys. Rept. 349 (2001) 125 [astro-ph/0010468] [INSPIRE].

[25] M.F. Morales and J.B. Wyithe, Reionization and Cosmology with $21 \mathrm{~cm}$ Fluctuations, Ann. Rev. Astron. Astrophys. 48 (2010) 127 [arXiv:0910.3010] [INSPIRE].

[26] J.R. Pritchard and A. Loeb, 21-cm cosmology, Rept. Prog. Phys. 75 (2012) 086901 [arXiv: 1109.6012] [INSPIRE].

[27] A. Natarajan and N. Yoshida, The Dark Ages of the Universe and Hydrogen Reionization, PTEP 2014 (2014) 06B112 [arXiv: 1404.7146] [INSPIRE].

[28] Planck collaboration, Planck 2013 results. XVI. Cosmological parameters, Astron. Astrophys. 571 (2014) A16 [arXiv:1303.5076] [INSPIRE].

[29] SDSS collaboration, The discovery of a luminous $z=5.80$ quasar from the Sloan Digital Sky Survey, Astron. J. 120 (2000) 1167 [astro-ph/0005414] [INSPIRE].

[30] SDSS collaboration, Evidence for Reionization at $Z \sim 6$ : Detection of a Gunn-Peterson trough in a $Z=6.28$ Quasar, Astron. J. 122 (2001) 2850 [astro-ph/0108097] [INSPIRE].

[31] A. Ewall-Wice, T.-C. Chang, J. Lazio, O. Doré, M. Seiffert and R.A. Monsalve, Modeling the Radio Background from the First Black Holes at Cosmic Dawn: Implications for the $21 \mathrm{~cm}$ Absorption Amplitude, Astrophys. J. 868 (2018) 63 [arXiv:1803.01815] [INSPIRE].

[32] C. Feng and G. Holder, Enhanced global signal of neutral hydrogen due to excess radiation at cosmic dawn, Astrophys. J. Lett. 858 (2018) L17 [arXiv:1802.07432] [INSPIRE].

[33] J.R. Pritchard and S.R. Furlanetto, $21 \mathrm{~cm}$ fluctuations from inhomogeneous X-ray heating before reionization, Mon. Not. Roy. Astron. Soc. 376 (2007) 1680 [astro-ph/0607234] [INSPIRE].

[34] A. Mesinger, S. Furlanetto and R. Cen, 21cmFAST: A Fast, Semi-Numerical Simulation of the High-Redshift 21-cm Signal, Mon. Not. Roy. Astron. Soc. 411 (2011) 955 [arXiv:1003.3878] [INSPIRE].

[35] E. Visbal, R. Barkana, A. Fialkov, D. Tseliakhovich and C. Hirata, The signature of the first stars in atomic hydrogen at redshift 20, Nature 487 (2012) 70 [arXiv:1201.1005] [INSPIRE].

[36] H. Tashiro and N. Sugiyama, The effect of primordial black holes on $21 \mathrm{~cm}$ fluctuations, Mon. Not. Roy. Astron. Soc. 435 (2013) 3001 [arXiv:1207.6405] [INSPIRE].

[37] A. Mesinger, A. Ferrara and D.S. Spiegel, Signatures of X-rays in the early Universe, Mon. Not. Roy. Astron. Soc. 431 (2013) 621 [arXiv:1210.7319] [INSPIRE].

[38] F. Pacucci, A. Mesinger, S. Mineo and A. Ferrara, The X-ray spectra of the first galaxies: 21 cm signatures, Mon. Not. Roy. Astron. Soc. 443 (2014) 678 [arXiv:1403.6125] [INSPIRE].

[39] A. Fialkov, R. Barkana and E. Visbal, The observable signature of late heating of the Universe during cosmic reionization, Nature 506 (2014) 197 [arXiv: 1402.0940] [INSPIRE]. 
[40] R. Ghara, T.R. Choudhury and K.K. Datta, $21 \mathrm{~cm}$ signal from cosmic dawn: imprints of spin temperature fluctuations and peculiar velocities, Mon. Not. Roy. Astron. Soc. 447 (2015) 1806 [arXiv: 1406.4157] [INSPIRE].

[41] A. Fialkov, A. Cohen, R. Barkana and J. Silk, Constraining the redshifted 21-cm signal with the unresolved soft X-ray background, Mon. Not. Roy. Astron. Soc. 464 (2017) 3498 [arXiv:1602.07322] [INSPIRE].

[42] J. Raste and S. Sethi, An Analytic Formulation of the $21 \mathrm{~cm}$ Signal from the Early Phase of the Epoch of Reionization, Astrophys. J. 860 (2018) 55 [arXiv:1711.03827] [InSPIRE].

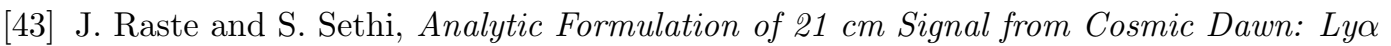
Fluctuations, Astrophys. J. 876 (2019) 56 [arXiv:1810.12746] [INSPIRE].

[44] P. Madau, A. Meiksin and M.J. Rees, 21-CM tomography of the intergalactic medium at high redshift, Astrophys. J. 475 (1997) 429 [astro-ph/9608010] [INSPIRE].

[45] P. Tozzi, P. Madau, A. Meiksin and M.J. Rees, The first light seen in the redshifted 21-cm radiation, Nucl. Phys. B Proc. Suppl. 80 (2000) 0509 [astro-ph/9905199] [InSPIRE].

[46] N.Y. Gnedin and P.A. Shaver, Redshifted $21 \mathrm{~cm}$ emission from the pre-reionization era 1 . Mean signal and linear fluctuations, Astrophys. J. 608 (2004) 611 [astro-ph/0312005] [INSPIRE].

[47] S.K. Sethi, HI signal from reionization epoch, Mon. Not. Roy. Astron. Soc. 363 (2005) 818 [astro-ph/0508172] [INSPIRE].

[48] M. Zaldarriaga, S.R. Furlanetto and L. Hernquist, 21 Centimeter fluctuations from cosmic gas at high redshifts, Astrophys. J. 608 (2004) 622 [astro-ph/0311514] [INSPIRE].

[49] S. Furlanetto, M. Zaldarriaga and L. Hernquist, The growth of HII regions during reionization, Astrophys. J. 613 (2004) 1 [astro-ph/0403697] [INSPIRE].

[50] S. Furlanetto, M. Zaldarriaga and L. Hernquist, Statistical probes of reionization with $21 \mathrm{~cm}$ tomography, Astrophys. J. 613 (2004) 16 [astro-ph/0404112] [INSPIRE].

[51] J.D. Bowman, A.E.E. Rogers, R.A. Monsalve, T.J. Mozdzen and N. Mahesh, An absorption profile centred at 78 megahertz in the sky-averaged spectrum, Nature 555 (2018) 67 [arXiv: 1810.05912] [INSPIRE].

[52] R. Barkana, Possible interaction between baryons and dark-matter particles revealed by the first stars, Nature 555 (2018) 71 [arXiv: 1803.06698] [INSPIRE].

[53] G. Lambiase and S. Mohanty, Hydrogen spin oscillations in a background of axions and the 21-cm brightness temperature, Mon. Not. Roy. Astron. Soc. 494 (2020) 5961 [arXiv: 1804.05318] [INSPIRE].

[54] S. Fraser et al., The EDGES $21 \mathrm{~cm}$ Anomaly and Properties of Dark Matter, Phys. Lett. B 785 (2018) 159 [arXiv: 1803.03245] [INSPIRE].

[55] J.B. Muñoz and A. Loeb, A small amount of mini-charged dark matter could cool the baryons in the early Universe, Nature 557 (2018) 684 [arXiv:1802.10094] [INSPIRE].

[56] B.T. Draine and J. Miralda-Escudé, Absorption by Spinning Dust: A Contaminant for High-redshift $21 \mathrm{~cm}$ Observations, Astrophys.J.Lett. 858 (2018) L10 [arXiv:1804.02250].

[57] J. Chluba, Green's function of the cosmological thermalization problem - II. Effect of photon injection and constraints, Mon. Not. Roy. Astron. Soc. 454 (2015) 4182 [arXiv: 1506.06582] [INSPIRE].

[58] S.A. Wouthuysen, On the excitation mechanism of the 21-cm (radio-frequency) interstellar hydrogen emission line., Astron. J. 57 (1952) 31.

[59] G.B. Field, The spin temperature of intergalactic neutral hydrogen, Astrophys. J. 129 (1959) 536. 
[60] G.B. Field, Excitation of the Hydrogen 21-CM Line, Proceedings of the IRE 46 (1958) 240.

[61] X.-L. Chen and J. Miralda-Escudé, The Spin-Kinetic Temperature Coupling and the Heating Rate due to Lya Scattering before Reionization: Predictions for 21 Centimeter Emission and Absorption, Astrophys. J. 602 (2004) 1 [astro-ph/0303395] [INSPIRE].

[62] G.B. Field, The Time Relaxation of a Resonance-Line Profile, Astrophys. J. 129 (1959) 551.

[63] G.B. Rybicki and I.P. Dell'Antonio, The time development of a resonance line in the expanding universe, Astrophys. J. 427 (1994) 603 [astro-ph/9312006] [inSPIRE].

[64] P.A. Shaver, R.A. Windhorst, P. Madau and A.G. de Bruyn, Can the reionization epoch be detected as a global signature in the cosmic background?, Astron. Astrophys. 345 (1999) 380 [astro-ph/9901320] [INSPIRE].

[65] P. Sharma, Astrophysical radio background cannot explain the EDGES 21-cm signal: constraints from cooling of non-thermal electrons, Mon. Not. Roy. Astron. Soc. 481 (2018) L6 [arXiv: 1804.05843] [INSPIRE].

[66] R. Hills, G. Kulkarni, P.D. Meerburg and E. Puchwein, Concerns about modelling of the EDGES data, Nature 564 (2018) E32 [arXiv: 1805.01421] [INSPIRE].

[67] A.S. Kompaneets, The Establishment of Thermal Equilibrium between Quanta and Electrons, JETP 4 (1957) 730.

[68] R. Sunyaev and Y.B. Zeldovich, The interaction of matter and radiation in the hot model of the universe, II, Astrophys. Space Sci. 7 (1970) 20.

[69] Y.B. Zel'dovich, A. Illarionov and R. Syunyaev, The effect of energy release on the emission spectrum in a hot universe, JETP $\mathbf{3 5}$ (1972) 643.

[70] A. Illarionov and R. Siuniaev, Comptonization, the spectrum of relict radiation, and the thermal history of the universe, Astron. Zh. 51 (1974) 1162.

[71] L. Pozdnyakov, I. Sobol and R. Sunyaev, Comptonization and the shaping of X-ray source spectra - Monte Carlo calculations, Sov. Sci. Rev. E 2 (1983) 189.

[72] L. Danese and G. de Zotti, Double Compton process and the spectrum of the microwave background, Astron. Astrophys. 107 (1982) 39.

[73] J. Chluba, S. Sazonov and R.A. Sunyaev, The double Compton emissivity in a mildly relativistic thermal plasma within the soft photon limit, Astron. Astrophys. 468 (2007) 785 [astro-ph/0611172] [INSPIRE].

[74] G. Rybicki and A. Lightman, Radiative processes in astrophysics, WILEY-VCH Verlag GmbH \& Co. KGaA (1979).

[75] Y.B. Zel'Dovich and E. Levich, Stationary state of electrons in a nonequilibrium radiation field, ZhPmR 11 (1970) 57.

[76] E. Levich and R. Sunyaev, The heating of gas in the vicinity of quasars, nuclei of seyfert galaxies, and pulsars by the induced compton effect, Astrophys. Lett. 7 (1970) 69.

[77] Y.B. Zel'Dovich and E. Levich, Bose condensation and shock waves in photon spectra, Sov. Phys. JETP 28 (1969) 1287.

[78] A. Illarionov and R. Syunyaev, Compton scattering by thermal electrons in x-ray sources., Sov. Astron. 16 (1972) 45.

[79] R. Syunyaev, Induced compton scattering by thermal electrons and the low-frequency spectrum of radio sources., Sov. Astron. 15 (1971) 190.

[80] J. Arons, Radiative Transfer of Isotropic X-Rays and Gamma Rays. II. High-Energy Radiation in the Expanding Universe, Astrophys. J. 164 (1971) 457. 
[81] J. Bernstein and S. Dodelson, Aspects of the Zeldovich-sunyaev Mechanism, Phys. Rev. D 41 (1990) 354 [INSPIRE].

[82] M. Seiffert et al., Interpretation of the ARCADE 2 Absolute Sky Brightness Measurement, Astrophys. J. 734 (2011) 6.

[83] M. Pospelov, J. Pradler, J.T. Ruderman and A. Urbano, Room for New Physics in the Rayleigh-Jeans Tail of the Cosmic Microwave Background, Phys. Rev. Lett. 121 (2018) 031103 [arXiv: 1803.07048] [INSPIRE].

[84] S. Dodelson, Modern Cosmology, Academic Press (2003). 